\title{
Follicular cholangitis associated with focal biliary stricture treated with left hepatectomy after 8 years of follow-up: A rare case report
}

\author{
RYUSUKE SAITO ${ }^{1}$, TOSHIKATSU FUKUDA ${ }^{1}$, HIRONOBU AMANO ${ }^{1}$, MASAHIRO NAKAHARA ${ }^{1}$, \\ MAKOTO YOSHIDA $^{1}$, MINORU YAMAKI ${ }^{1}$, KEIJI HANADA ${ }^{2}$, SHUJI YONEHARA $^{3}$ and TOSHIO NORIYUKI ${ }^{1,4}$ \\ Departments of ${ }^{1}$ Surgery, ${ }^{2}$ Gastrointestinal Medicine and ${ }^{3}$ Pathology, \\ Onomichi General Hospital, Onomichi, Hiroshima 722-8508; ${ }^{4}$ Department of Gastroenterological \\ and Transplant Surgery, Hiroshima University, Hiroshima 734-8551, Japan
}

Received May 27, 2015; Accepted October 13, 2015

DOI: $10.3892 / \mathrm{mco} .2015 .659$

\begin{abstract}
Follicular cholangitis may lead to severe focal biliary stricture, which is difficult to distinguish from cholangiocarcinoma. This is the report of a rare case of follicular cholangitis associated with an intrahepatic focal biliary stricture in a 69-year-old woman. The patient visited our hospital for upper abdominal pain in 2006. Computed tomography revealed common bile duct stones and focal stricture of the left intrahepatic duct. Endoscopic retrograde cholangiopancreatography revealed a severe B3 stricture and dilation of the peripheral biliary tract. Repeated cytology revealed no malignancy. The patient was followed up for 8 years and repeated attacks of cholangitis severely compromised her quality of life. A left hepatectomy was performed, and the pathological findings were consistent with follicular cholangitis. It is generally difficult to make a definitive diagnosis after identifying a biliary stricture, and the stricture is often treated with surgical resection. The clinical presentation and radiological findings of follicular cholangitis are similar to those of cholangiocarcinoma; therefore, if a conservative approach is selected, frequent follow-up is recommended. The present case suggests that follicular cholangitis should be considered in the differential diagnosis of biliary stricture.
\end{abstract}

\section{Introduction}

Follicular cholangitis is difficult to diagnose, as it is particularly challenging to distinguish from primary sclerosing cholangitis, immunoglobulin G4 (IgG4)-related sclerosing cholangitis and hilar cholangiocarcinoma (1-4). Follicular cholangitis is relatively rare; it was first reported by Aoki et al (5) in 2003 , and only a few cases have been reported to date (5-9). Imaging

Correspondence to: $\mathrm{Dr}$ Toshio Noriyuki, Department of Surgery, Onomichi General Hospital, 1-10-23 Hirahara, Onomichi, Hiroshima 722-8508, Japan

E-mail: nori0509_0217@yahoo.co.jp

Key words: follicular cholangitis, hepatectomy, hepatolithiasis, case report, focal biliary strictures findings representative of this disease include biliary stricture associated with dilatation of the peripheral bile duct and lymph node enlargement. Certain patients have undergone surgical treatment for preoperative diagnosis of cholangiocarcinoma, which was finally diagnosed as a benign condition (10).

It was previously demonstrated that the majority of the cases are diagnosed as cholangiocarcinoma due to the severe biliary stricture and the presence of symptoms, which commonly include abdominal pain and jaundice. Follicular cholangitis has recently been reported to be among several diseases that cause biliary stricture. The reported pathological findings of this disease are fibrotic thickening of the wall of the hilar bile duct; prominent hyperplastic lymph follicles with germinal centers in the subserosal layer, without tumorous growth, which were considered to be reactive hyperplastic follicles, with localization of the lesions in the proximal extrahepatic bile duct and hepatic hilum, and with almost intact peripheral intrahepatic bile ducts (8).

We herein report the case of a 69-year-old woman with follicular cholangitis associated with a focal biliary stricture, who underwent left hepatectomy after 8 years of follow-up.

\section{Case report}

A 69-year-old woman was referred to our hospital due to repeated hepatolithiasis and cholangitis of the left hepatic lobe over an 8-year follow-up period. The patient was $148 \mathrm{~cm}$ in height and weighed $36.5 \mathrm{~kg}$, had undergone abdominal surgery, including hysterectomy for myoma and a laparoscopic cholecystectomy 7 years earlier, and had no history of autoimmune disease. The laboratory data revealed no elevation in the levels of hepatobiliary enzymes or tumor markers, such as carcinoembryonic antigen and carbohydrate antigen 19-9. Internal drainage to the B3 using endoscopic retrograde biliary drainage had been performed annually for cholangitis. Endoscopic treatment for cholangitis had also been repeatedly performed due to obstruction of the tube. On abdominal ultrasonography, there was dilatation of the left intrahepatic duct to a width of $\leq 2 \mathrm{~cm}$, and stones with an acoustic shadow. Computed tomography (CT) and magnetic resonance cholangiopancreatography (MRCP) revealed severe stricture of the left intrahepatic biliary duct and dilatation of the peripheral 

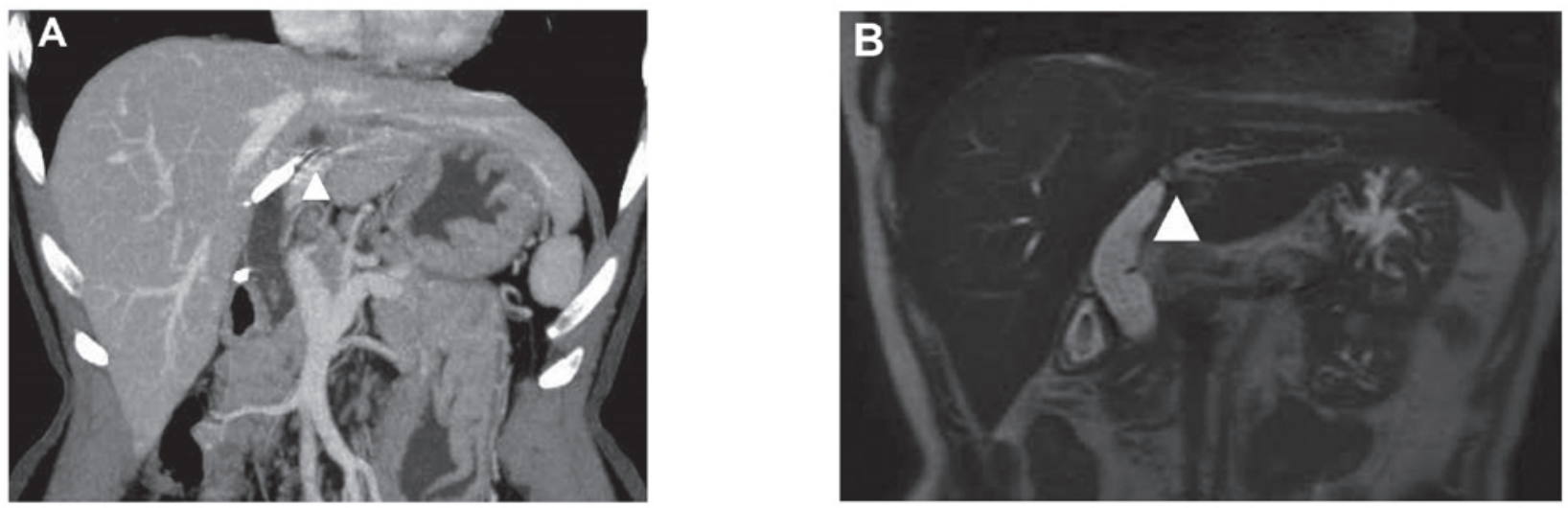

Figure 1. (A) Computed tomography and (B) magnetic resonance cholangiopancreatography revealed a severe stricture of the left intrahepatic biliary duct (arrowheads), with dilatation of the peripheral biliary duct.

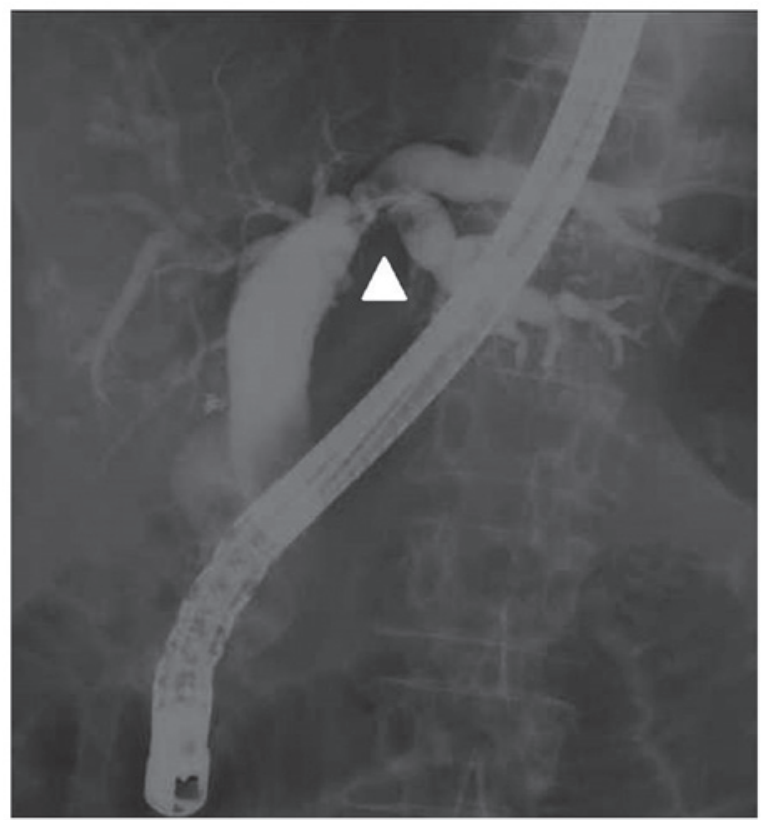

Figure 2. Endoscopic retrograde cholangiopancreatography demonstrated a severe stricture of the left intrahepatic biliary duct with dilatation of the peripheral biliary duct, which was consistent with the findings of the magnetic resonance cholangiopancreatography.

biliary duct (Fig. 1). Tumors or enlarged lymph nodes were not identified. A drainage tube was inserted into the B3 and small stones were identified in the common bile duct. Endoscopic retrograde cholangiopancreatography (ERCP) revealed a severe stricture of the left intrahepatic biliary duct and dilatation of the peripheral biliary duct, which was consistent with the MRCP findings (Fig. 2). A left hepatectomy was performed. No mass lesions were detected around the liver hilum intraoperatively; however, a focal bile stricture was observed in the left liver hilum. Macroscopically, inflamed granulation tissue was only found around the thickened biliary duct. There was no mass lesion around the liver hilum; however, the wall of the duct was markedly thickened (Fig. 3). Microscopically, there was dense fibrosis under the mucosal layer, which was accompanied by marked formation of lymph follicles containing germinal centers. The peribiliary glands were infiltrated by lymphocytes and plasma cells. No obliterative phlebitis

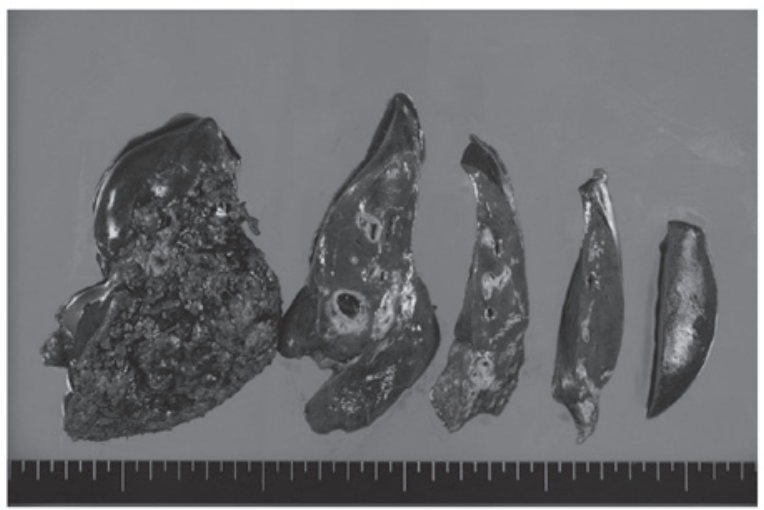

Figure 3. There were no macroscopically evident tumors in the hepatic hilum in this specimen, although the thickness of the wall was markedly increased.

or vasculitis were observed. There were few IgG4-positive cells (Fig. 4). The pathological findings were consistent with follicular cholangitis. The patient did not experience recurrence of cholangitis postoperatively for 1 year.

\section{Discussion}

Making a precise diagnosis upon identifying a biliary stricture is difficult, and distinguishing a benign stricture from malignant disease may be particularly challenging. Follicular cholangitis is extremely rare and, to the best of our knowledge, only 9 cases have been reported to date since Aoki et al (5) first described this condition in 2003. A review of previous reports on follicular cholangitis is presented in Table I. The radiological findings commonly indicate biliary stricture, which mimics hilar cholangiocarcinoma, and there are no specific symptoms or radiological findings that may be used to exclude malignancy. The only ways to diagnose this disease are histopathological analysis, or observation over an extended period of time in order to determine whether it is progressive.

Representative histological findings have shown that follicular cholangitis is characterized by dense fibrosis under the mucosal layer, which is accompanied by marked formation of lymph follicles that contain germinal centers. The peribiliary glands are infiltrated by lymphoplasmacytic cells that are negative for IgG4 but positive for CD20, B-cell lymphoma 2 

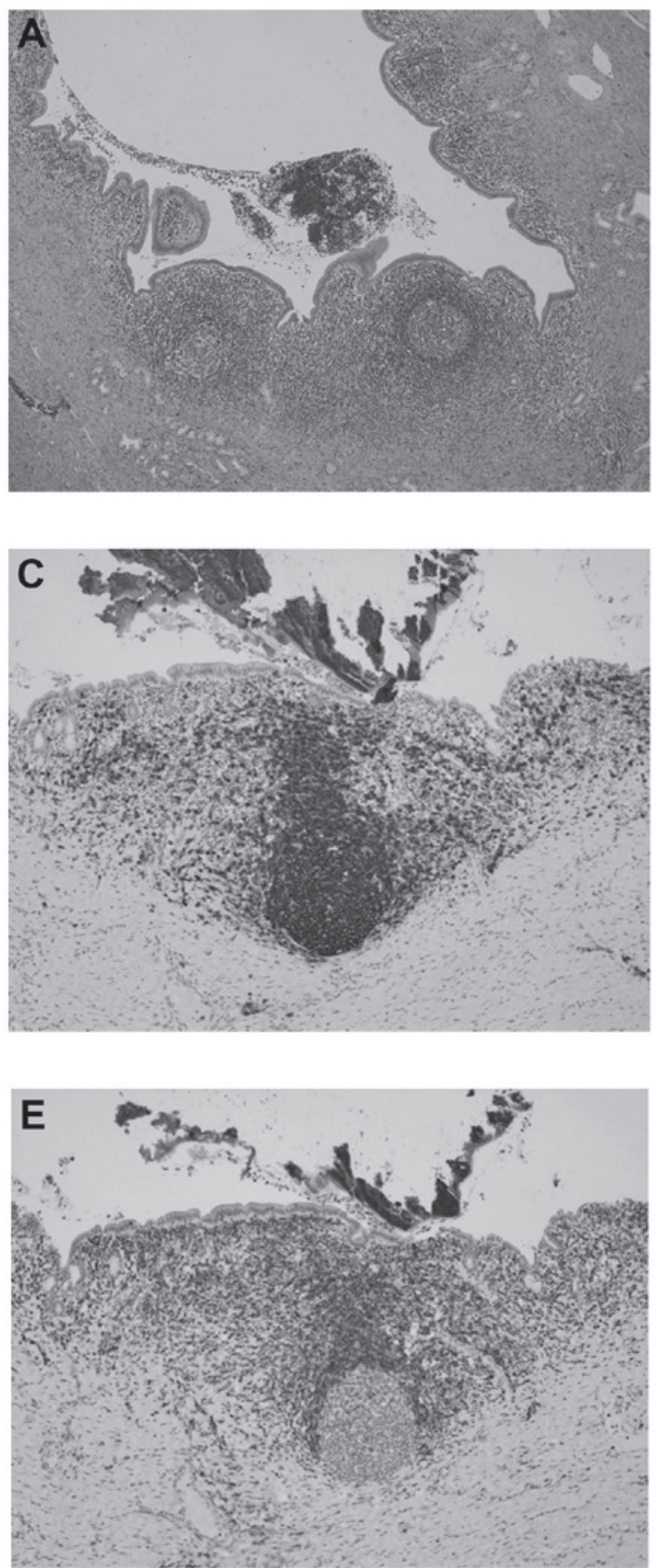
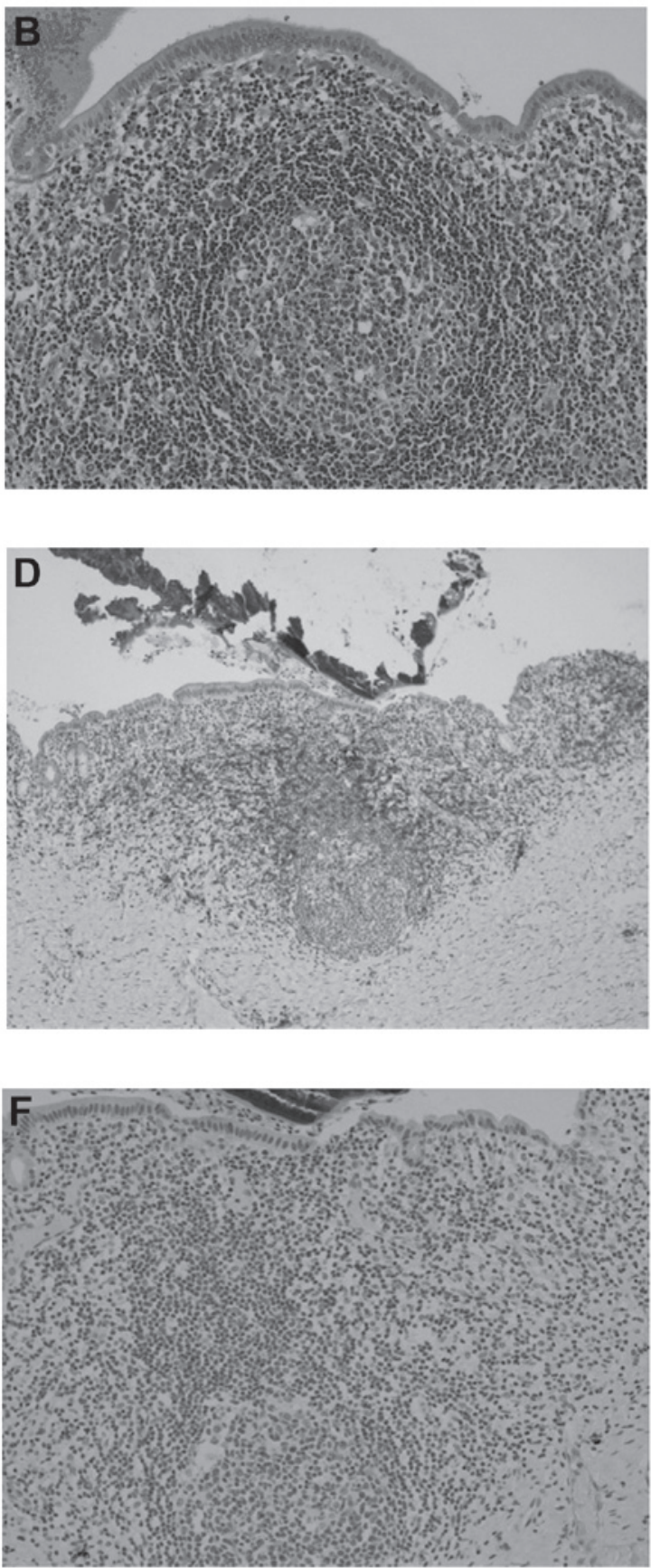

Figure 4. Hematoxylin and eosin staining; magnification, (A) x5 and (B) x20. The wall of the bile duct was markedly thickened; dense fibrosis was observed under the mucosal layer, accompanied by marked formation of lymph follicles that contained germinal centers. Immunohistochemical staining for (C) CD20: magnification, x10; (D) CD3: magnification, x10; (E) B-cell lymphoma 2 (Bcl2): magnification, x10; and (F) immunoglobulin G4 (IgG4): magnification, x20. The peribiliary glands are infiltrated by lymphocytes and plasma cells, positive for CD20, Bcl2 and CD4, but negative for IgG4.

and CD4. These pathological findings, rather than the radiological findings and clinical presentation, are the only ones to definitively diagnose follicular cholangitis. Our pathological findings were consistent with those reported for follicular cholangitis. Fujii et al (9) described the clinical presentation and the step-by-step diagnosis of this disease. Follicular cholangitis commonly occurs during middle age and in patients with no significant history. The majority of the patients presented with a gradual progression of the disease; the focal stricture was localized to the hilum of the liver; the majority of the patients underwent surgical treatment for suspicion of cholangiocarcinoma. A total of 2 patients experienced recur- rence of cholangitis; 1 succumbed to liver failure, whereas the remaining patient was treated with corticosteroids and achieved a moderate improvement with close follow-up (6).

This is the first case in which the patient finally underwent hepatectomy due to repeated attacks of cholangitis after 8 years of follow-up. We were able to identify the stricture of the root of the left biliary duct on presentation in 2006. However, the decision for surgical resection was based on the symptomatic hepatolithiasis. The indications for hepatectomy for hepatolithiasis are as follows: Hepatolithiasis in the unilateral lobe, severe stricture or dilatation of the biliary duct, hepatolithiasis associated with intrahepatic bile duct carcinoma, accompanied 


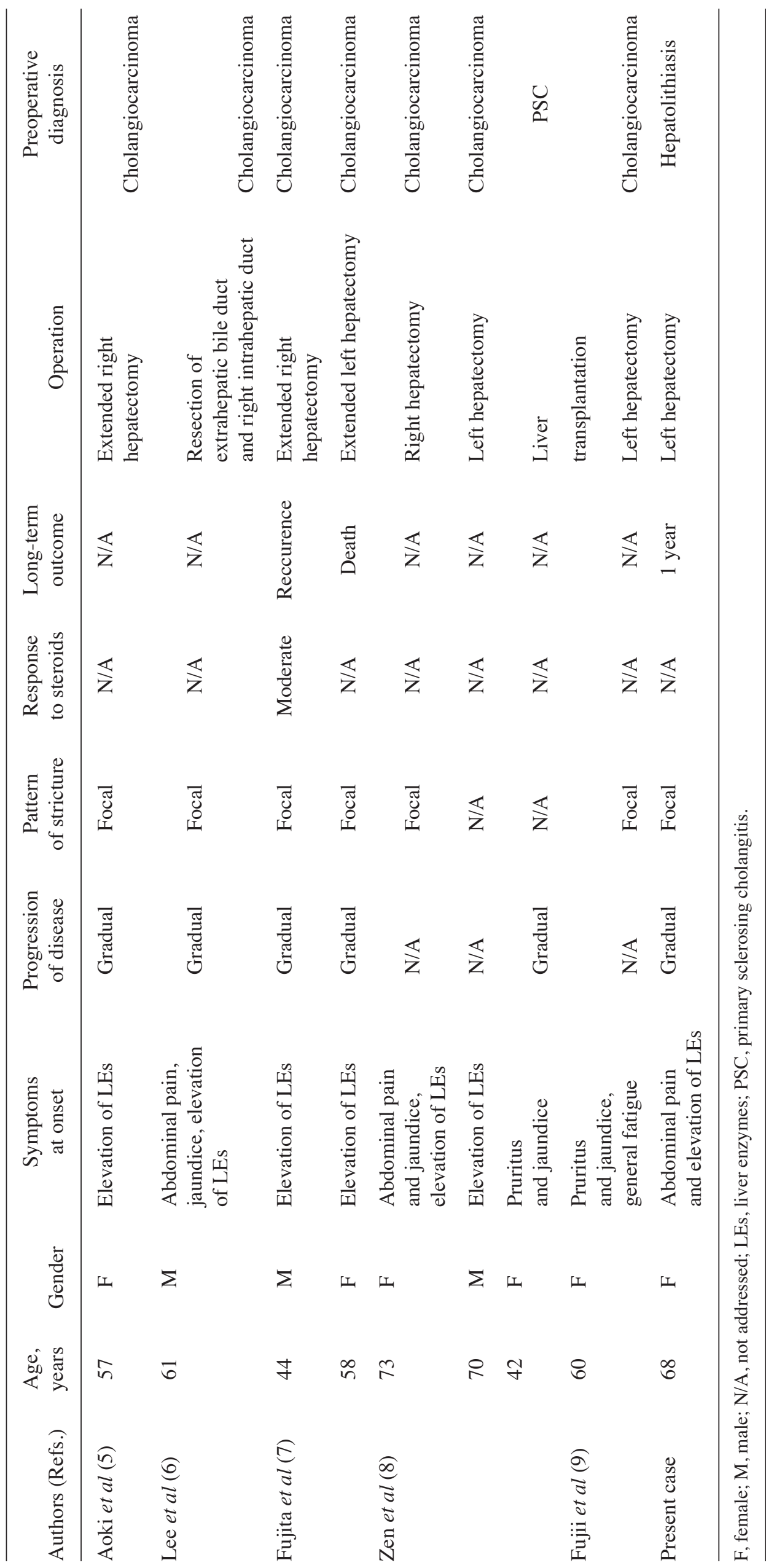


by formation of hepatic abscesses or atrophy of the liver $(11,12)$. The radiological findings mimicked those of intrahepatic cholangiocarcinoma; however, repeated cytology of the bile juice revealed no malignancy. Following close radiological follow-up, lymph node enlargement or progressive biliary stricture were not observed. Therefore, conservative treatment was selected. Over this long-term follow-up period, there were no findings on ERCP and CT indicating progression.

This case suggests that close follow-up is recommended if a conservative approach is selected following identification of a focal biliary stricture, since its clinical presentation and radiological findings are similar to those of cholangiocarcinoma.

Herein, we presented a case of follicular cholangitis with a focal intrahepatic stricture. It is difficult to differentiate follicular cholangitis from hilar cholangiocarcinoma; thus, surgical treatment should be performed if close follow-up is not possible. Clinicians must consider causes of benign biliary stricture, such as follicular cholangitis, which are indistinguishable from malignancy.

\section{References}

1. Tamada K, Kanai N, Wada S, Tomiyama T, Ohashi A, Satoh Y, Ido K and Sugano K: Utility and limitations of intraductal ultrasonography in distinguishing longitudinal cancer extension along the bile duct from inflammatory wall thickening. Abdom Imaging 26: 623-631, 2001.

2. van Leeuwen DJ and Reeders JWAJ: Primary sclerosing cholangitis and cholangiocarcinoma as a diagnostic and therapeutic dilemma. Ann Oncol 10 (Suppl 4): 89-93, 1999.

3. Lee YM and Kaplan MM: Primary sclerosing cholangitis. N Engl J Med 332: 924-933, 1995.
4. Hamano H, Kawa S, Uehara T, Ochi Y, Takayama M, Komatsu K, Muraki T, Umino J, Kiyosawa K and Miyagawa S: Immunoglobulin G4-related lymphoplasmacytic sclerosing cholangitis that mimics infiltrating hilar cholangiocarcinoma: Part of a spectrum of autoimmune pancreatitis? Gastrointest Endosc 62: 152-157, 2005

5. Aoki T, Kubota K, Oka T, Hasegawa K, Hirai I and Makuuchi M: Follicular cholangitis: Another cause of benign biliary stricture. Hepatogastroenterology 50: 639-642, 2003.

6. Lee JY, Lim JH and Lim HK: Follicular cholangitis mimicking hilar cholangiocarcinoma. Abdom Imaging 30: 744-747, 2005.

7. Fujita T, Kojima M, Kato Y, Gotohda N, Takahashi S, Konishi M and Kinoshita T: Clinical and histopathological study of 'follicular cholangitis': Sclerosing cholangitis with prominent lymphocytic infiltration masquerading as hilar cholangiocarcinoma. Hepatol Res 40: 1239-1247, 2010.

8. Zen Y, Ishikawa A, Ogiso S, Heaton $\mathrm{N}$ and Portmann B: Follicular cholangitis and pancreatitis - clinicopathological features and differential diagnosis of an under-recognized entity. Histopathology 60: 261-269, 2012.

9. Fujii M, Shiode J, Niguma T, Ito M, Ishiyama S, Fujiwara A, Nose S, Yoshioka M and Mimura T: A case of follicular cholangitis mimicking hilar cholangiocarcinoma. Clin J Gastroenterol 7: 62-67, 2014.

10. Gerhards MF, Vos P, van Gulik TM, Rauws EAJ, Bosma A and Gouma DJ: Incidence of benign lesions in patients resected for suspicious hilar obstruction. Br J Surg 88: 48-51, 2001.

11. Uchiyama K, Onishi H, Tani M, Kinoshita H, Ueno M and Yamaue H: Indication and procedure for treatment of hepatolithiasis. Arch Surg 137: 149-153, 2002.

12. Chijiiwa K, Yamashita H, Yoshida J, Kuroki S and Tanaka M: Current management and long-term prognosis of hepatolithiasis. Arch Surg 130: 194-197, 1995. 